\title{
ASO Visual Abstract: Predicting Colon Cancer-Specific Survival for the Asian Population Using National Cancer Registry Data from Taiwan
}

Han-Ching Chan, MS ${ }^{1}$, Chi-Cheng Huang, MD, PhD ${ }^{2,3}$, Ching-Chieh Huang, MS ${ }^{1}$, Amrita Chattopadhyay, PhD $^{1}$, Kuan-Hung Yeh, BS ${ }^{2}$, Wen-Chung Lee, MD, PhD ${ }^{1,4}$, Chun-Ju Chiang, $\mathrm{PhD}^{1,4}$, Hsin-Ying Lee, $\mathrm{MS}^{1}$, Skye Hung-Chun Cheng, $\mathrm{MD}^{5}$, and Tzu-Pin $\mathrm{Lu}, \mathrm{PhD}^{1}$ (i)

${ }^{1}$ Department of Public Health, Institute of Epidemiology and Preventive Medicine, College of Public Health, National Taiwan University, Taipei, Taiwan; ${ }^{2}$ Department of Public Health, College of Public Health, National Taiwan University, Taipei, Taiwan; ${ }^{3}$ Division of General Surgery, Department of Surgery, Taipei Veterans General Hospital, Taipei, Taiwan; ${ }^{4}$ Taiwan Cancer Registry, Taipei, Taiwan; ${ }^{5}$ Department of Radiation Oncology, Koo Foundation Sun Yat-Sen Cancer Center, Taipei, Taiwan

This study proposes a prediction model that provides an accurate estimation of prognosis for colon cancer patients and accurately predicts colon cancer-specific survival, regardless of patient ethnicity (https://doi.org/10.1245/s10 434-021-10646-2). The model can assist treatment decisions for patients from diverse ethnic backgrounds.

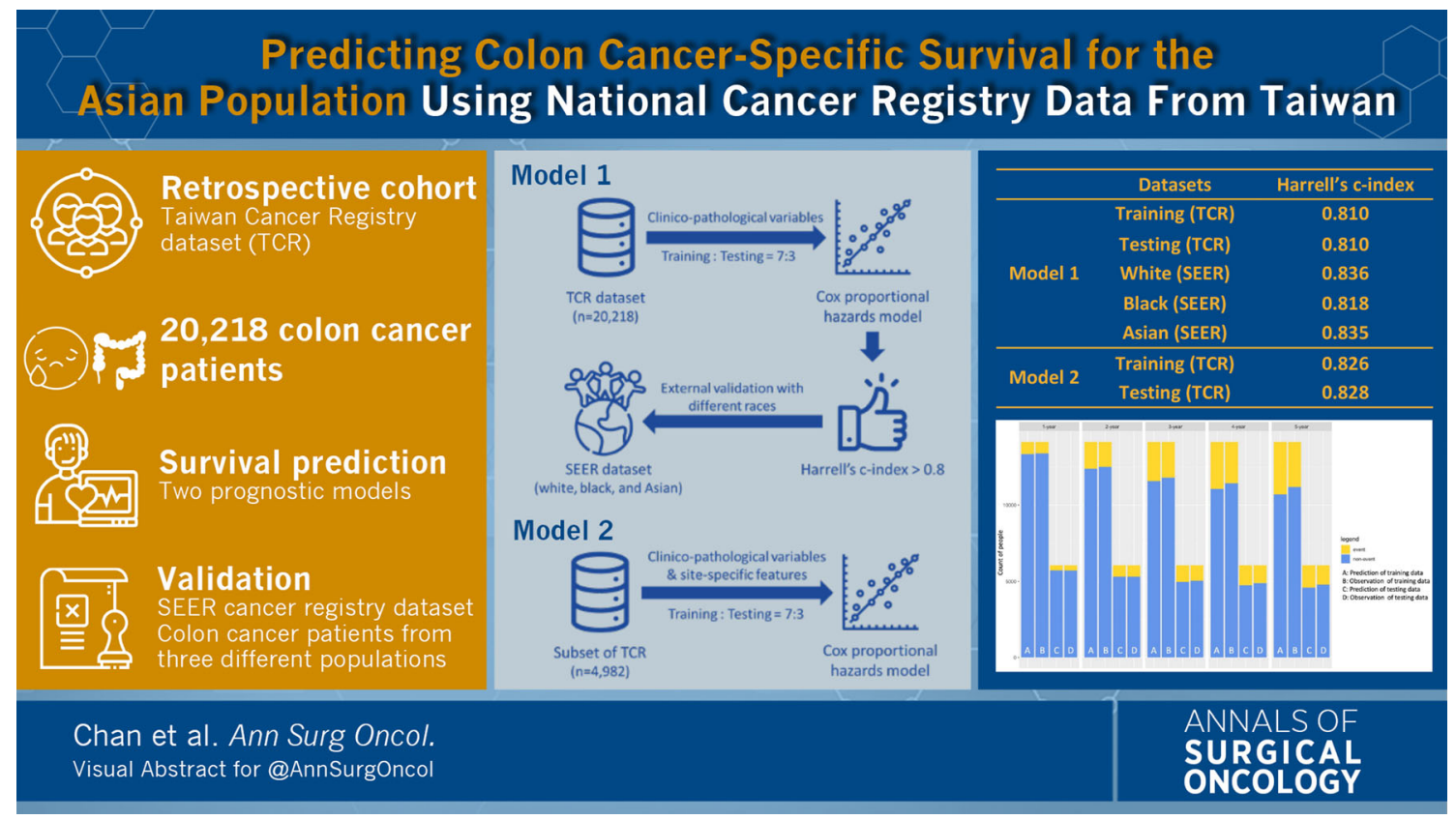

(C) Society of Surgical Oncology 2021

Published Online: 25 August 2021

T.-P. Lu, PhD

e-mail: tplu@ntu.edu.tw
DISCLOSURE There are no conflicts of interest.

Publisher's Note Springer Nature remains neutral with regard to jurisdictional claims in published maps and institutional affiliations. 